\title{
Sitagliptin induced splenic infarcts
}

\section{ABSTRACT}

Sitagliptin belongs to the class of dipeptidyl peptidase-4 (DPP-4) inhibitors. DPP-4 inhibitors are remarkably well tolerated oral antihyperglycemic drugs with an adverse effect profile similar to that of placebo. No adverse effects have been reported even with overdose of sitagliptin in suicide attempts. We present here case summary of a 35-year-old female with type 2 diabetes mellitus and major depressive disorder who presented with multiple splenic infarcts after ingestion of sitagliptin $700 \mathrm{mg}$ and metformin $14 \mathrm{~g}$ with suicidal intent. To the best of our knowledge, there are no published reports of splenic infarcts as an adverse event of sitagliptin. (Clin Diabetol 2017; 6, 6: 215-217)

Key words: dipeptidyl peptidase-4 inhibitors, sitagliptin, splenic infarcts

\section{Introduction}

Sitagliptin belongs to the class of dipeptidyl peptidase-4 (DPP-4) inhibitors. DPP-4 inhibitors are remarkably well tolerated oral antihyperglycemic drugs with an adverse effect profile similar to that of placebo [1]. The other advantages with this class include that they are weight neutral and hypoglycemia is rare [1]. Although the currently available DPP-4 inhibitors are thought to be highly selective, continued long-term surveillance for

\section{Address for correspondence:}

Dr. Raiz Ahmad Misgar

Department of Endocrinology

Sher-i-Kashmir Institute of Medical Sciences

Srinagar, Kashmir, India

Phone: 09419090026

Fax: +911942403470

e-mail: drreyaz07@rediffmail.com

Clinical Diabetology 2017, 6, 6, 215-217

DOI: 10.5603/DK.2017.0035

Received: 11.11 .2017

Accepted: 08.01.2018 unexpected adverse events is essential. The multiplicity of substrates and peptide fragments whose biology DPP-4 inhibitors affect imparts complexity to the biology of these agents [2].

We present here case summary of a 35-year-old female with type 2 diabetes mellitus (T2DM) and major depressive disorder who presented with multiple splenic infarcts after ingestion of sitagliptin $700 \mathrm{mg}$ and metformin $14 \mathrm{~g}$ with suicidal intent (7 times greater than the approved maximum daily dose of sitagliptin and over 5 times the approved maximum daily dose of metformin). To the best of our knowledge, there are no published reports of splenic infarcts as an adverse event of sitagliptin.

\section{Case study}

A 35-year-old normotensive female, known to have T2DM for last 11 years and major depressive disorder presented with severe left upper quadrant pain accompanied by pleuritic chest pain and left shoulder pain of few days duration. The pain was associated with nausea and vomiting. She was conscious and alert. Her blood pressure was 140/80 mm Hg. Abdominal examination revealed left upper quadrant tenderness. Her medications included 2 doses of premixed insulin, a fixed - dose combination pill of sitagliptin $50 \mathrm{mg}$ and metformin $1000 \mathrm{mg}$, levothyroxine $50 \mu \mathrm{g}$ and escitalopram $20 \mathrm{mg}$. She had been on sitagliptin for last 2 years and on insulin for last 8 months. She had poor glycemic control as reflected by $\mathrm{HbA}_{1 \mathrm{c}}$ of $8.5 \%$ and fasting plasma glucose of $200 \mathrm{mg} / \mathrm{dL}$ and postprandial glucose of $320 \mathrm{mg} / \mathrm{dL}$. Baseline investigations including complete blood counts, hematocrit, ESR, kidney and liver function tests were unremarkable; total platelet count was $2.2 \mathrm{lacs} / \mathrm{mm}^{3}$. Serum amylase was normal. Ultrasonography abdomen was normal. Pain was managed with analgesics and she was advised to report back in case of persistence/worsening of symptoms. Patient reported back two days later with worsening of pain. On further revision of history, she admitted that 

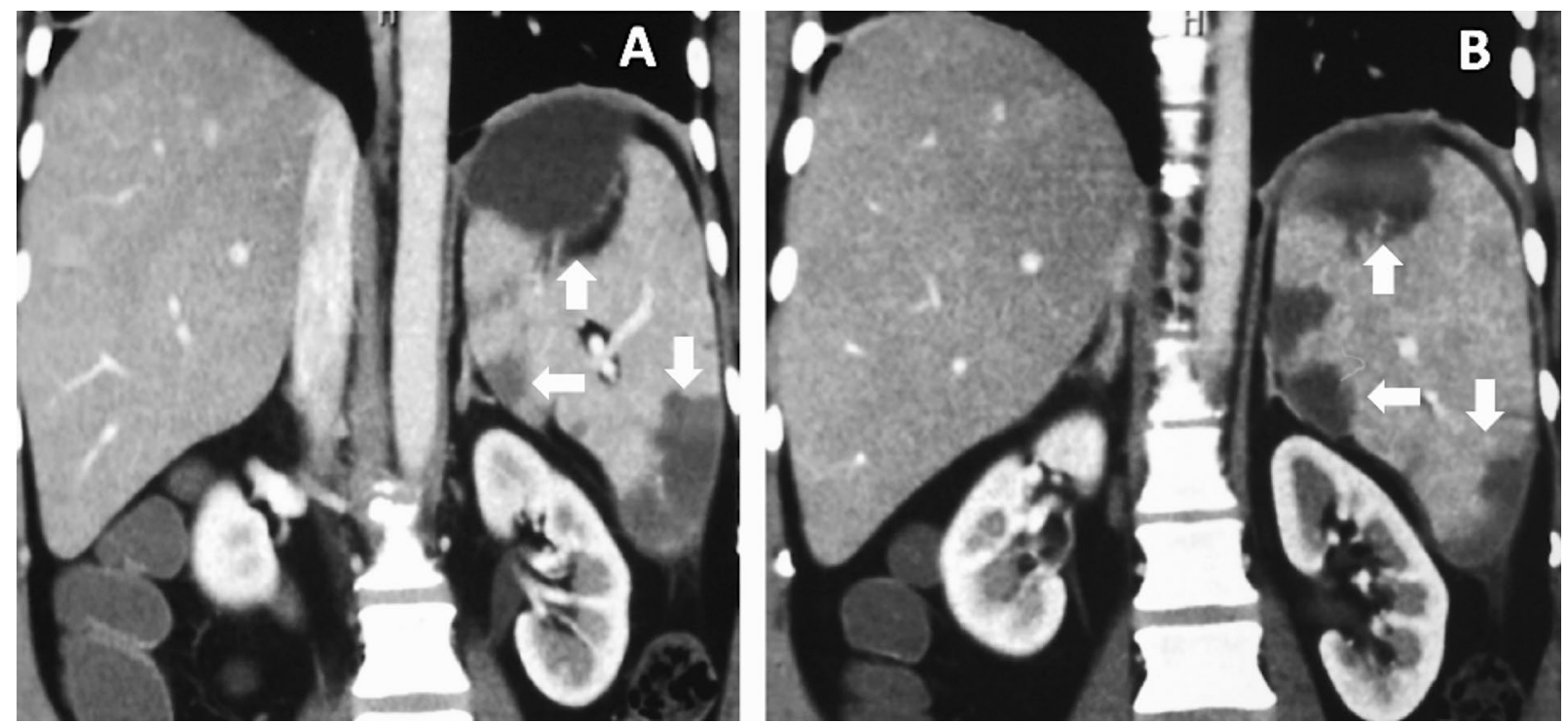

Figure 1. Contrast enhanced computed tomography of the abdomen showing multiple splenic infarcts (arrows)

she had taken 14 fixed-dose combination pills containing sitagliptin $50 \mathrm{mg}+$ metformin $1000 \mathrm{mg}$ each, amounting to a total ingestion of sitagliptin $700 \mathrm{mg}$ and metformin $14 \mathrm{~g}$ with suicidal intent one day prior to the development of symptoms.

For further evaluation of her symptoms, contrast enhanced computed tomography (CECT) of the abdomen was performed which revealed multiple fairly well defined hypodense lesions in spleen without any significant enhancement suggestive of splenic infarcts with bilateral minimal basal atelectasis (Fig. 1A and B). CECT also revealed minimal fluid and fat strandings posteroinferior to spleen. Serum $\mathrm{pH}$ was normal as was serum lactate levels. A detailed clinical and investigative work up was undertaken to find any possible etiology of splenic infarcts. There was no clinical suggestion of the presence of underlying hematological, thromboembolic or collagen vascular disease. The patient had never taken estrogens. Echocardiography did not reveal any evidence of valvular lesion or endocarditis. The D-dimer test and antinuclear antibody were negative. Coagulogram was normal. Serum protein C, protein S, homocysteine levels were normal. The serology for hepatitis $C$ was negative. The work up for anti-phospholipid antibody syndrome (lupus anticoagulant, antiphospholipid antibodies IgG and IgM, dilute Russell Viper Venom test) was negative.

A clear temporal relation between ingestion of "large doses" of sitagliptin and development of splenic infarcts together with negative results in the work up for any known etiologies of splenic infarct strongly incriminate sitagliptin as the cause for splenic infarcts in our patient. Patient was taken off sitagliptin and the other medications were continued. Her management included hydration, analgesics, and frequent monitoring. After few days her symptoms abated and she was completely asymptomatic a week later. A repeat CECT abdomen could not be undertaken as the patient declined it.

\section{Discussion}

The adverse effect profile DPP-4 inhibitors is similar to that of placebo and no adverse effects have been reported even with overdose of DPP-4 inhibitors [3, 4]. Furukawa et al., reported no adverse effects in a 86-year-old woman with type 2 diabetes and depression, after ingestion of 1,700 mg of sitagliptin (17 times greater than the approved maximum daily dose) with suicidal intent [3]. In another reported case, an overdose of sitagliptin $3500 \mathrm{mg}$ (35 times greater than the approved maximum daily dose) did not lead to any adverse events [4]. This is the highest reported case for sitagliptin overdose. These two published reports reflect that "large doses" of sitagliptin do not lead to any adverse events. Our patient developed splenic infarcts with $700 \mathrm{mg}$ of sitagliptin; this may reflect individual vulnerability to an adverse event with this drug.

An eHealthMe based on reports of 41.491 persons who had side effects with sitagliptin, updated on $25^{\text {th }}$ Jul, 2017 from FDA, reported splenic infarction in 13 persons $(0.03 \%)$ [5]. Splenic infarction was reported especially in males, those who had been taking the drug for 2 to 5 years, with concomitant use of exenatide and having essential hypertension. In this report all patients were 60 years of age or older [5].

The mechanism of sitagliptin induced splenic infarcts is unknown. Thrombosis may be induced with 
inhibition of DPP-4 activity. DPP-4, also known as CD26, is expressed by microvascular endothelial cells in humans (i.e. in liver, spleen, lungs and brain) and in the hearts of rats $[6,7]$. DPP-4 has the potential to inhibit fibrin polymerization and clot formation $[8,9]$. Thus DPP-4 may behave as an immobilized anti-coagulant on microvascular endothelium and possesses antithrombotic properties; decreased DPP-4 expression and activity has been found to coincide with an increase in Tissue Factor expression and induction of in situ thrombosis [10]. Inhibition of DPP-4 activity also induces adhesion of platelets; this may be another mechanism of thrombosis which occurs with inhibition of DPP-4 activity [10]. For two reasons we strongly believe that metformin was not responsible for splenic infarcts in our patient. Firstly, metformin has never been reported to cause splenic infarction or infarction of any other viscera. Secondly, metformin has not been reported to have any unfavourable effect on fibrin polymerization, coagulation and platelets.

Our patient was a young female, had been on sitagliptin for 2 years only, was normotensive and had never been on exenatide. To the best of our knowledge this is the first published case report of sitagliptin induced splenic infarcts. Our case highlights that abdominal pain in a patient on sitagliptin may indicate the presence of splenic infarcts and deserves thorough evaluation and management. In addition our case emphasizes that splenic infarcts due to sitagliptin phosphate may occur in young individuals without other risk factors like essential hypertension and the concomitant use of exenatide.

\section{Conflict of interest}

The authors report no conflicts of interest.

\section{REFERENCES}

1. Deacon CF, Lebovitz HE, Deacon CF. Dipeptidyl peptidase-4 inhibitors in the treatment of type 2 diabetes: a comparative review. Diabetes Obes Metab. 2011; 13(1): 7-18, doi: 10.1111/j.1463-1326.2010.01306.x, indexed in Pubmed: 21114598.

2. Ussher JR, Drucker DJ. Cardiovascular actions of incretin-based therapies. Circ Res. 2014; 114(11): 1788-1803, doi: 10.1161/ /CIRCRESAHA.114.301958, indexed in Pubmed: 24855202.

3. Furukawa S, Kumagi T, Miyake T, et al. Suicide attempt by an overdose of sitagliptin, an oral hypoglycemic agent: a case report and a review of the literature. Endocr J. 2012; 59(4): 329-333, doi: 10.1507/endocrj.ej11-0390, indexed in Pubmed: 22277726.

4. Sehra S, Jaggi S, Sehra D, et al. Management of Sitagliptin and Metformin Combination Toxic Overdose. J Assoc Physicians India. 2016; 64(11): 80-81, indexed in Pubmed: 27805342.

5. Januvia and Splenic infarction - from FDA reports. http://www. ehealthme.com/ds/januvia/splenic-infarction/;25th July 2017 (30th July 2017)

6. Gorrell MD. Dipeptidyl peptidase IV and related enzymes in cell biology and liver disorders. Clin Sci (Lond). 2005; 108(4): 277-292, doi: 10.1042/CS20040302, indexed in Pubmed: 15584901.

7. Koyama T, Gao M, Ueda T, et al. Different enzyme activities in coronary capillary endothelial cells. Adv Exp Med Biol. 1997; 411: 359-364, doi: 10.1007/978-1-4615-5865-1_45, indexed in Pubmed: 9269449.

8. Heymann E, Mentlein R. A negative blood-clotting factor lining the vessels. Naturwissenschaften. 1982; 69(4): 189-191, doi: 10.1007/bf00364899, indexed in Pubmed: 7045702.

9. Mentlein R, Heymann E. Dipeptidyl peptidase IV inhibits the polymerization of fibrin monomers. Arch Biochem Biophys. 1982; 217(2): 748-750, doi: 10.1016/0003-9861(82)90556-2, indexed in Pubmed: 6753751

10. Krijnen PAJ, Hahn NE, Kholová I, et al. Loss of DPP4 activity is related to a prothrombogenic status of endothelial cells: implications for the coronary microvasculature of myocardial infarction patients. Basic Res Cardiol. 2012; 107(1): 233, doi: 10.1007/ /s00395-011-0233-5, indexed in Pubmed: 22167343. 